\title{
Research on Electrical Discharge Machining Intelligent Control Arithmetic
}

\author{
Hudai $\mathrm{Fu}^{1, \mathrm{a}}$, Jingang $\mathrm{Gao}^{2, \mathrm{~b}}$, Weiwei $\mathrm{Li}^{3, \mathrm{c}}$ \\ ${ }^{1,2,3}$ School of Mechatronics Engineering, Changchun Institute of Technology, Changchun, JiLin, \\ 130012, China \\ a,b,c email: fuhudai@126.com
}

Keywords: EDM; portable; intelligent control; Simulation

\begin{abstract}
A new vibration device of process electrode has been provided in the paper, which achieves the aim of developing process state and improving process speed. Set out from improving system process efficiency and process quality, it analyses the characteristics of EDM and make sure appropriate parameter and control system configuration. Combined the characteristics of fuzzy controller and artificial nerve net, it designs portable EDM nerve fuzzy control system, which uses in adjusting the parameter of servo control system and revises some disadvantages of faulty rule to improve quick respond capacity of control system. Applying fuzzy theory and simulation means, it analyses the portable EDM servo control system and proves the stability of the EDM control system in theory. Through typical experiments, the process efficiency of device has been testified.
\end{abstract}

\section{Introduction}

EDM technology is not only an important part of advanced manufacturing, but also a crucial supplement and development of machine cutting and grinding in machine manufacturing. With the development of EDM technology in the new century, the requirement of maneuverability, machining efficiency and machining quality for EDM machine is higher and higher. Intelligent control, as a new theory, has gradually become a hot research in EDM fields and provided an efficient way in order to solve the problem of control during complex process. The new outputs produced in this way have brought large economic benefits [1][2].

\section{Putting forward EDM nerve fuzzy control arithmetic}

The physical processing of EDM is moment and complex. The micro-procedure of each electrical discharge erosion is the integrative process of electric field force, electromagnetic force, thermic power, electrochemistry and colloid chemistry. But it is impossible to build a precise mathematic model at present. So we can not complete the micro control of discharge machining, namely the control for each pulse discharge capability.

The present EDM control system evaluate discharge state from the point of view of macroscope. First statistically analyze micro variable and get macro judgement standard. Second synthetically estimate multifold macro inspection variables. Third only use single macro-variable to control. The method will be applied when the requirement of machining quality is low.

A better way is to use fuzzy control for this kind of problem. We can build fuzzy control rule and express it in the form of fuzzy language, by using of some characteristic of mathematic model for the controlled object. Then we can get the exact conclusion, based on the subjection input in the fuzzy congregation and fuzzy rules produced by experience [3].

\section{Design for EDM fuzzy controller}

(1) Fuzzilization of input variable

Firstly, control system check space current through checking circuit during EDM. Secondly comparing it with optimal average current $I_{r}$, we can get input variable, namely space current 
error $^{E(t)}$. Third we can get another input variable by calculating error change rate ${ }^{E c(t)}$. Lastly transform these two precise variables into fuzzy variables.

$$
\begin{aligned}
& E(t)=I(t)-I_{r} \\
& E c(t)=E(t)-E(t-1)
\end{aligned}
$$

The above $I(t)$ is the discharge space current when the time of ${ }^{t}$. $I_{r}$ is the optimal discharge current and unchangeable during EDM. So:

$$
E c(t)=I(t)-I(t-1)
$$

The range of $E(t)$ and $E c(t)$ is $[-4,+4]$ in the control system. The discrete domain is $\{-4,-3$, $-2,-1,0,+1,+2,+3,+4\}$. We separate domain into five parts, $\widetilde{E}=\{P L, P M, Z E, N M, N L\}$ $\widetilde{E} C=\{P L, P M, Z E, N M, N L\}$ and adopt triangle subjection function to transform the input variables.

We can calculate according to the following formula when subjection range is $[\alpha, \beta]$ and subjection function is triangle.

Fuzzilization of output variable:

The output of control system is the regulate quantity $\Delta I(t)$ to space current value. $\Delta I(t)$ is positive when space current is smaller. Otherwise, $\Delta I(t)$ is negative.

(2) Building up fuzzy controlling rule library and fuzzy reasoning rule library:

In EDM fuzzy controlling, rule library consists of experience knowledge which makes discharge machining in the optimal discharge state. Controlling rule describes the knowledge. According to EDM experience knowledge, the corresponding controlling rules which include 25 items.

Because the fuzzy subsets of $\tilde{E}_{\text {and }} \tilde{E} c$ are both 5this table includes as many rule numbers as possible. Under common condition, the rule numbers can be less than 25 and the relevant column can be blank.

Suppose known inputs are $E\left(t_{0}\right)$ and $E c\left(t_{0}\right)$, fuzzy operation adopts single pot fuzzy set .So the corresponding input fuzzy sets are $\mathrm{A}^{\prime}$ and $B^{\prime}$ :

$$
\begin{aligned}
& \mu_{A^{\prime}}\left(E\left(t_{0}\right)\right)= \begin{cases}1 & \mathrm{E}\left(\mathrm{t}_{0}\right)=x_{0} \\
0 & E\left(t_{0}\right) \neq x_{0}\end{cases} \\
& \mu_{\mathrm{B}^{\prime}}\left(E c\left(t_{0}\right)\right)= \begin{cases}1 & \mathrm{Ec}\left(\mathrm{t}_{0}\right)=y_{0} \\
0 & \mathrm{Ec}\left(\mathrm{t}_{0}\right) \neq y_{0}\end{cases}
\end{aligned}
$$

(3) Clear up fuzzy variable

The reasoning result of fuzzy controller is fuzzy variable, namely a fuzzy subset. It can not be used to control practically and must be dealt with. So we can get a precise value reflecting fuzzy variable. The process will be named clearance.

Using barycenter arithmetic to do antifuzzilization which can gain clear variable. We can calculate the whole possible combinations in advance, because the amount of system input and output domain elements is finite. When practically controlling, we can merely check controlling rule table[4][5].

\section{Bring forward EDM fuzzy nerve control arithmetic}

Fuzzy nerve control system combined by fuzzy control and Neural Network is one of the most attentive researching aspects. The merit of both is the model inadequately depends on controlled object. Fuzzy Neural Network controller is composed of fuzzy arithmetic and one Neural Network. During machining, fuzzy controller simulates uncertain decision action by adopting fuzzy reasoning rule. But it must automatically produce rule from experience and the revision function of control 
decision self-study is not perfect. The introduction of Neural Network makes fuzzy control capability guideline better. So it is likely for fuzzy nerve control system to be deeply studied and widely applied during EDM.

\section{Design for EDM fuzzy Neural Network controller}

During EDM, the introduction of fuzzy nerve controller can greatly increase machining quality and efficiency of machine tool. EDM servocontrol system as follows is mainly composed of checking system, fuzzy nerve controller, servo system and EDM machine tool.

Fuzzy Neural Network controller is the core of the control system and its network structure is showed in Figure1.

The first layer is the system input layer for the sake of fuzzy controller to input parameter language variable. It includes $m=10$ nodes. Each network input cell corresponds to some or other fuzzy subset of input variable $E(t), E c(t)$.

The second layer is network crytic layer in which each node represents one fuzzy rule. Thus it requires that the number of nodes in crytic layer is more than that of fuzzy control rule. The third layer is network output layer. Each network output cell corresponds to value quantitied in output variable space domain. Because servo reference current changed-value $\Delta I$ is disparted into 13 parts ,namely $\{-6,-5,-4,-3,-2,-1,0,+1,+2,+3,+4,+5,+6\}$, the number of network output nodes is $n=13$.

We can train Neural Network by using of 25 fuzzy control rules mentioned above till the error between network output value and expected output value is small enough or zero. Thus once leaning is over. Fuzzy control rules have transformed network weight and inlayed into the Neural Network, after 25 rules have been entirely trained [6].

\section{Experiment study of EDM system}

It shows that the physics essential of ED from point of view of ED space volt-ampere character by using of the portable EDM machine expounded in the paper. It expects that the macro physics value, voltage and current which affect the micro discharge process would tightly combine macro operation and micro response. It will show up the real reason of affecting ED physics essential. Consequently it will reflect the realizablity of EDM system.

Experiment project: Remove the aiguille and wimble broken in the workpiece.

When drilling small hole and using wimble to process screw thread, the aiguille and wimble will be broken in the workpiece for the reason of rigid and fragile tool and low antiflexibility and antiwrest. In order to avoid discarding workpiece, we can remove the aiguille and wimble broken in the workpiece by using EDM.

Electrode diameter should be determined based on the dimension of aiguille and wimble. The diameter of aiguille tool electrode showed in Figure 2 (a): $d 0 \leq d^{\prime} \leq \mathrm{d}$.Commonly d0 is about (1/5) $\mathrm{d}$. So we can assume electrode diameter $d^{\prime}=(2 / 5 \sim 4 / 5) \mathrm{d}$. When processing screw thread, $\mathrm{d} 0 \leq$ $d^{\prime} \leq \mathrm{d}, \quad d^{\prime}=\frac{d_{0}+d}{2} \quad$, Figure 2 (b) shows it..

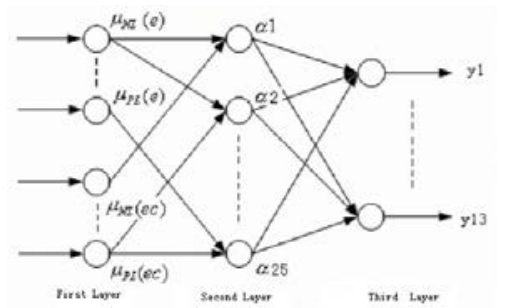

Fig.1. Discharge machining fuzzy Neural Network

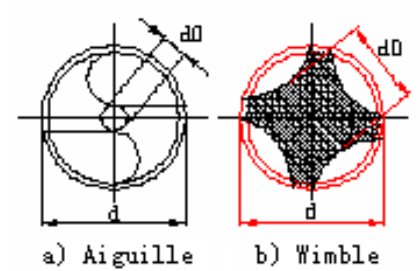

Fig.2. aiguille and wimble section 
We fix the electrode prepared into electrode collet and plumb the electrode to machine tool work table-board. Then we fix the workpiece on the EDM machine work table-board and move table-board so as to make electrode center and aiguille or wimble center which has been broken in the workpice consistent. Last we put it into machining fluid waiting for processing.

The experiment results are showed in Figure 3(a), (b), and (c).
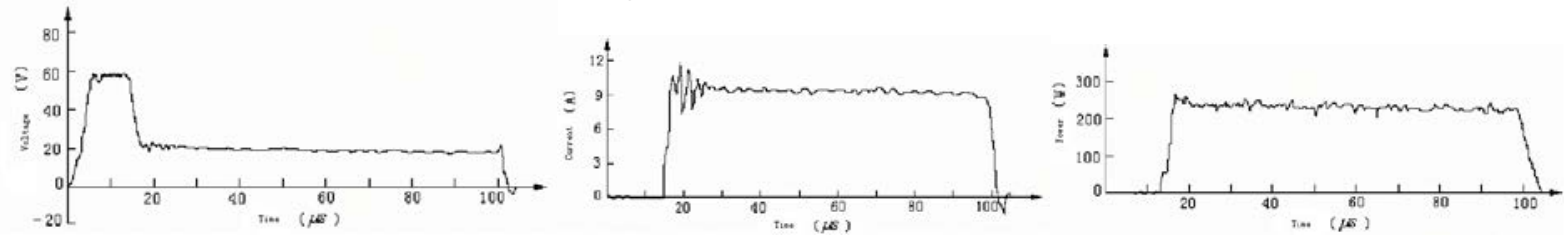

(a) Square wave pulse ED voltage wave (b) Square wave pulse ED current wave wave pulse ED power wave

(c) Square

Fig.3. Square wave pulse ED voltage, current and power wave

Power output voltage will go down to zero when it reaches square wave voltage pulse keeping time of power output. The additional voltage between space two ends will rapidly decline to zero from keeping voltage. Space current will fall rapidly. Because of the effect of the inductance in space, space voltage will fall slowly.

Space voltage will decline rapidly when space current passes zero in the next time. At last space voltage, current and power become zero. The whole discharge process finishes. The discharge space removes the ionization. Another discharge machining will begin when the next pulse arrives.

\section{Conclusion}

It pursuits EDM machine which is efficient, stable, low-price and runs agilely. The traditional EDM machine not only has huge volume, high price, but also brings a lot inconveniences to the machining industries. It has systematically studied EDM machine structure, process intelligent control system structure, intelligent control arithmetic, the stability of control system and so on.

\section{References}

[1]K. Kobayashi. The Present and Future Developments of EDM and ECM Proc [J]. ISEM XI Switzerland, 1995. 14(135) 29-47.

[2]G.V.Saiu, u. Adaptive Hierarchical Fuzzy Controller [J]. IEEE Tans SMC, 1993.23(4) 160-161.

[3]S.I.Horikawa. et al. On Design of Adaptive Fuzzy Controller Using Fuzzy Sets and systems, 2008. (3) 53-55.

[4]Eckart Uhlmann, Sascha Piltz, Kai Schauer. Micro Milling of Sintered Tungsten-copper Composite Materials [J]. Journal of Materials Processing Technology, 2005. (167) 402-407.

[5]W. Wang, S.H. Kweon, S.H. Yang. A Study on Roughness of the Micro-end-milled Surface Produced by a Miniatured Machine Tool [J]. Journal of Material Processing Technology, 2005. (162) 702-708.

[6]M.T. Zaman, A. Senthil Kumar, M. Rahman, S. Sreeram. A Three-Dimensional Analytical Cutting Force Model for Micro End Milling Operation [J]. International Journal of Machine Tool \& Manufacture, 2006. (460) 353-366.

[2] Xiling He. Force analysis of the elastic sleeve structure [J]. Hoisting and Conveying Machinery, 2005. (3) 36-37. 